\title{
Relationship between Genetic Polymorphisms in MTHFR (C677T, A1298C and their Haplotypes) and the Incidence Of Breast Cancer among Jordanian Females - Case-Control Study
}

\author{
Nemah Awwad*,Al-Motassem Yousef,Ali Abuhaliema, Ihab Abdalla, Muhammad \\ Yousef
}

\begin{abstract}
Background: Breast cancer is a major cause of morbidity and mortality in Jordan and worldwide. Abnormality of DNA methylation is a possible mechanism for the development of cancer. Methylenetetrahydrofolate reductase (MTHFR) is involved in DNA methylation. Our aim was to study the association between genetic polymorphisms of MTHFR at two sites (C677T and A1298C) and their haplotypes and the risk of breast cancer among Jordanian females. Materials and Methods: A case-control study involving 150 breast cancer cases and 150 controls was conducted. Controls were age-matched to cases. Polymerase chain reaction/restriction fragment length polymorphism (PCR-RFLP) technique and sequencing were conducted to determine the genotypes. Results: There was a significant difference in genotype frequency of $\mathrm{C677T}$ in the 41-60 year age category [cases: CC

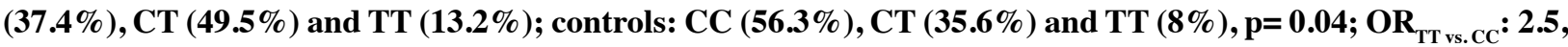

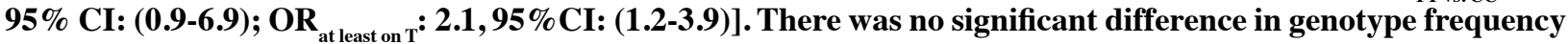
of A1298C between cases and controls [cases: AA (46.6\%), AC (41.8\%) and CC (11.6\%); controls: AA (43\%), AC $(47.4 \%)$ and $\mathrm{CC}(\mathbf{9 . 6 \%}) ; \mathrm{p}=\mathbf{0 . 6}$. There was a significant difference of MTHFR genetic polymorphism haplotypes among breast cancer cases and controls [cases/control: $\mathrm{CA}: 38.3 / 45.4 \% ; \mathrm{CC}: 28.9 / 25.2 \% ; \mathrm{TA:}$ 29.2/21; TC: $3.6 / 8.3 ; p$ value $\left.=0.01 ; \mathrm{OR}_{\mathrm{TA} \text { vs. } \mathrm{CA}}=1.6 ; 95 \% \mathrm{CI}(1.1-2.5) ; \mathrm{p}=0.02\right]$. Conclusions: Genetic polymorphism of MTHFR C677T may modulate the risk of breast cancer especially in the 41-60 year age group. Additionally, TA haplotype amends the risk of breast cancer. Future studies with a larger sample size are needed to validate the role of MTHFR genetic polymorphisms in breast cancer.
\end{abstract}

Keywords: Jordanian females - MTHFR - polymorphism - breast cancer - risk factor

Asian Pac J Cancer Prev, 16 (12), 5007-5011

\section{Introduction}

Cancer is a disease that is characterized by uncontrolled cellular growth, local tissue, and regional lymph nodes invasion as well as distant metastases (Chad M. Barnett et al., 2014). Ductal carcinoma is the most common type of breast cancer, which begins in the lining of the milk ducts (Butterworth et al., 2006). Another type of breast cancer is lobular carcinoma, which starts in the lobules (milk glands) of the breast.

In Jordan, breast cancer ranked first among cancers in females, accounting for $37.4 \%$ of all female cancers, and is the leading cause of cancer deaths among Jordanian women (Jordan ministry of health, 2010). According to the Jordan National Cancer Registry (JNCR) latest statistics, 978 cases of breast cancer in both sexes were newly diagnosed with breast cancer in 2010, which accounts for $19.8 \%$ of the total new cancer cases (Jordan ministry of health, 2010).
Although family history of breast cancer is common in women diagnosed with breast cancer, only less than $10 \%$ of all of the breast cancer cases are associated with inherited genetic mutations (Foulkes, 2008). Women who have a family history of breast cancer have a twofold higher lifetime risk of breast cancer than the general population (Pharoah et al., 1997) and a specific predisposing gene is identified in less than $30 \%$ of cases (Shiovitz and Korde, 2015).

The 5,10-Methylenetetrahydrofolate reductase (MTHFR) locus is located on chromosome 1 at the end of the short arm (1p36.6) (Gaughan et al., 2000). This enzyme is important in folate metabolism which is an essential process for cell metabolism in the DNA, RNA and protein methylation (Liew and Gupta, 2015). The protein which is encoded by this gene catalyzes the conversion of 5,10-methylenetetrahydrofolate to 5-methyltetrahydrofolate, which is a co-substrate for homocysteine remethylation to methionine $(\mathrm{Xu}$ et 
al., 2008). Genetic variation in this gene influences susceptibility to many types of cancer (Jiang et al., 2013; Ozen et al., 2014; Rai, 2014a; Yang et al., 2014). For example, MTHFR genetic polymorphisms have been linked to colorectal cancer (Yousef et al., 2013). Mutations in this gene will result in MTHFR deficiency (Frosst et al., 1995).

There are two commonly recognized polymorphic variants in the gene encoding for MTHFR: C677T (rs1801133, NC_000001.11:11796321, Ala222Val), and A1298C (rs1801133, NC_000001.11:11794419, Glu429Ala) variant; both are known to decrease the enzyme activity (Frosst et al., 1995). The mutation of the MTHFR gene which results in the C677T polymorphism is located at exon 4 which causes the conversion of valine to alanine at codon 222 , a common polymorphism that reduces the activity of this enzyme (Liew and Gupta, 2015). The second polymorphism in MTHFR gene is A1298C polymorphism results from $A$ to $C$ transition in exon 7 resulting in an amino acid substitution of glutamine to alanine at codon 429 of the protein.

The heterozygous mutated subjects of both MTHFR genetic polymorphisms have mildly raised homocysteine levels while the homozygous mutated subjects have higher homocysteine levels compared with the normal, non-mutated controls (Liew and Gupta, 2015).

In this case-control study, we studied the relationship between the genetic polymorphism of MTHFR C677T, MTHFR A1298C and their combined effect on the susceptibility of breast cancer among Jordanians.

\section{Materials and Methods}

\section{Study population}

A hospital-based study of 150 breast cancer women and 150 age-matched healthy individuals was conducted at Al-Basheer hospital/Amman which is a referral hospital for cancer. Study population were collected from the 15th of March till 21st of December 2014. The research was approved by the ethics committee in Al-Basheer hospital (institutional review board, IRB) in accordance with the ethical standards of the responsible committee on human experimentation (institutional and national) and with the Helsinki Declaration (IRB no. 17443, 28/11/2013). Each subject gave a sample of his/her blood after detailed explanation of the purpose of the study; a consent form was also obtained from each subject.

\section{MTHFR Genotyping}

Four milliliters of venous blood were collected from patients and healthy subjects in K3EDTA coated tubes. The tubes were kept in icebox and DNA extraction was performed in the same day using Wizard Genomic DNA purification kit (Promega Corporation, Wisconsin, USA) according to manufacturer's instructions. Amplification of the $\mathrm{C} 677 \mathrm{~T}$ region was performed using the forward primer ACTCAGCGAACTCAGCACTC (NC_000001.11: $11,796,104$ to $11,796,123)$ and the reverse primer AAGATCAGAGCCCCCAAAGC (NC_000001.11: $11,796,513$ to $11,796,494$ ) yielding a 410 -bp band, whereas for the A1298C polymorphism, the forward primer
GGGCTGTGAGTtGATGGTGA (NC_000001.11: $11,793,979$ to $11,793,998)$, and the reverse primer AGGAGCTGCTGAAGATGTG (NC_000001.11: $11,794,461$ to $11,794,443$ ), were used yielding a 483-bp band (Princess Haya Biotechnology Centre at the King Abdullah University Hospital, Jordan University for Science and Technology). The PCR conditions were: 4 minutes of initial denaturation at $95^{\circ} \mathrm{C}$, followed by 39 cycles of $95^{\circ} \mathrm{C}$ for 15 seconds, $60^{\circ} \mathrm{C}$ for 12 seconds, and $72^{\circ} \mathrm{C}$ for 15 seconds, with a final extension at $72^{\circ} \mathrm{C}$ for 10 minutes (Bio-Rad, S1000 Thermal cycler ${ }^{\mathrm{TM}}$, USA).

The PCR products of C677T were digested with HinfI restriction enzyme. Resulting fragments were visualized using RedSafe ${ }^{\mathrm{TM}}$ (New England Biolab, USA) staining and $2 \%$ agarose gel electrophoresis. The digestion fragment sizes for the C677T genotypes were: 384 and 26 bp bands for CC, 219,165, 26 bp bands for TT, and 384, 219, 165, 26 bp bands for CT.

Findings of the PCR-RFLP were validated by: 1) In every PCR run, a negative control was run simultaneously. A negative control contains all PCR components except the DNA template; 2) Around 33\% of all samples were repeated to confirm findings of the PCR-RFLP; 3) Randomly selected $10 \%$ PCR-RFLP results were confirmed by DNA sequencing using sanger didoxy method (Walker and Lorsch, 2013). The concordance between repeated samples, sequencing and our results was $100 \%$. The presence of an internal control which will be cut by HinfI irrespective of the genotype of C677T. The genotyping of A1298C was done by DNA sequencing for all samples [BigDye Terminator Cycle Sequencing on 3730xl DNA sequencer (Genewiz ${ }^{\circledR}$ Co., USA)].

\section{Haplotype analysis}

The interaction between genetic polymorphism at the two loci was assessed by evaluating the combinedgenotypes effects and haplotype analysis. We analyzed the haplotype frequencies of the two SNPs (C677T and A1298C) for breast cancer cases and compared them with those of controls. Haplotype frequencies were calculated using Multiallelic Interallelic Disequilibrium Analysis Software [(MIDAS $\left.{ }^{\circledR}\right)$, University of Southampton, Highfield, Southampton, UK (Tom R Gaunt et al., 2006)] and linkage disequilibrium was represented by Lewontin's coefficient (D').

\section{Statistical analysis}

Data were coded and entered into SPSS software version 16 (Chicago, IL). Data of categorical nature were summarized as counts and percentages. Data of continuous nature were summarized as mean \pm standard deviation. The relation between categorical $v s$. categorical variables were evaluated by Chi-square or Fisher exact test as appropriate. The strength of association was assessed by calculating odds ratio (OR) and $95 \%$ confidence interval (95\%CI) (Cochran G, 1954). The relationship between categorical variable and continuous variable was evaluated by independent t-test. A p value $<0.05$ was considered statistically significant. Hardy-Weinberg Equilibrium was assessed for genotypes and allele types (Rodriguez et al., 2009). 
Table 1. MTHFR C677T and MTHFR A1298C Genotypes and Allele Types and Risk of Breast Cancer

\begin{tabular}{|c|c|c|c|c|c|c|c|c|c|c|}
\hline \multirow[b]{2}{*}{ Age category } & \multicolumn{5}{|c|}{ C677T genetic polymorphism } & \multicolumn{5}{|c|}{ A1298C genetic polymorphism } \\
\hline & Genotypes & $\begin{array}{r}\text { Case (\%) } \\
\mathrm{N}=150\end{array}$ & $\begin{array}{c}\text { Control (\%) } \\
\mathrm{N}=146\end{array}$ & p value & $\overline{\mathrm{OR}}(95 \% \mathrm{CI})$ & Genotypes & $\begin{array}{r}\text { Case }(\%) \\
\mathrm{N}=146\end{array}$ & $\begin{array}{r}\text { Control (\%) } \\
\mathrm{N}=135\end{array}$ & $\mathrm{p}$ value & e OR $(95 \% \mathrm{CI})$ \\
\hline \multirow[t]{3}{*}{$24-40 \mathrm{yrs}$} & $\mathrm{CC}$ & $13(41.9)$ & $15(48.4)$ & $0.2 \mathrm{a}$ & Reference & AA & $12(40)$ & $13(43.3)$ & $0.9 \mathrm{a}$ & Reference \\
\hline & $\mathrm{CT}$ & $16(51.6)$ & $10(32.3)$ & & $1.9(0.6-5.5)$ & $\mathrm{AC}$ & $16(53.3)$ & $14(46.7)$ & & $1.24(0.43-3.58)$ \\
\hline & TT & $2(6.5)$ & $6(19.4)$ & & $0.4(0.07-2.3)$ & $\mathrm{CC}$ & $2(6.7)$ & $3(10.0)$ & & $0.72(0.10-5.1)$ \\
\hline \multirow[t]{3}{*}{$41-60 \mathrm{yrs}$} & $\mathrm{CC}$ & $34(37.4)$ & $49(56.3)$ & $0.04 \mathrm{~b}$ & Reference & AA & $42(47.7)$ & $38(48.1)$ & $0.4 \mathrm{~b}$ & Reference \\
\hline & $\mathrm{CT}$ & $45(49.5)$ & $31(35.6)$ & & $2.1(1.1-3.9)$ & $\mathrm{AC}$ & 34 (38.6) & $35(44.3)$ & & $0.88(0.46-1.7)$ \\
\hline & TT & $12(13.2)$ & $7(8)$ & & $2.5(0.9-6.9)$ & $\mathrm{CC}$ & $12(13.6)$ & $6(7.6)$ & & $1.81(0.62-5.3)$ \\
\hline \multirow[t]{3}{*}{$>60 \mathrm{yrs}$} & $\mathrm{CC}$ & 19 (67.9) & $15(53.6)$ & $0.5 \mathrm{a}$ & Reference & AA & $14(50.0)$ & $7(26.9)$ & $0.2 \mathrm{a}$ & Reference \\
\hline & $\mathrm{CT}$ & $8(28.6)$ & $10(35.7)$ & & $0.63(0.2-2)$ & $\mathrm{AC}$ & $11(39.3)$ & $15(57.7)$ & & $0.4(0.1-1.2)$ \\
\hline & TT & $1(3.6)$ & $3(10.7)$ & & $0.26(0.03-2.8)$ & $\mathrm{CC}$ & $3(10.7)$ & $4(15.4)$ & & $0.4(0.7-2.2)$ \\
\hline
\end{tabular}

*a: p value calculated by Fisher's exact test; b: p value calculated by chi square. OR: odds ratio; 95\%CI: 95\% Confidence interval (677: CC: homozygous wild type, CT: heterozygous, TT: homozygous mutant.) (1298: AA: homozygous wild type, AC: heterozygous, CC: homozygous mutant). Numbers don't add up to 150 due to unsuccessful PCR-RFLP product

\section{Results}

A total of 150 breast cancer patients and 150 control subjects were included in this study. Mean age in patients and control groups was $49.9 \pm 10.8$ years. No significant age differences were observed between the two arms $(\mathrm{p}=0.99)$. Median age at diagnosis for our cases was 49 years. Age at menarche and menopause were $13.5 \pm 1.6$ years and $49 \pm 4.6$ years, respectively.

The distribution of MTHFR C677T and A1298C genotypes is presented in Table 1. There were statistically significant differences in the genotype frequency of MTHFR C677T between cases and the controls in the age category of 41-60 years. The frequency of the MTHFR T allele was higher in patients with breast cancer compared with healthy controls. Subjects with the T allele were 1.7 time more likely to suffer from breast cancer $(\mathrm{p}=0.014)$. Carriers of the TT genotype have 2.5 times higher risk of breast cancer compared with carriers of CC genotype. This was not seen in patients " $<41$ years" and " $>60$ years" old. Also, the same results were found using "at least one $\mathrm{T}$ model" and allele frequencies. In age category of "41-60" years carriers of "at least one T" and " $\mathrm{T}$ allele" were at 1.5 and 1.75 times higher risk of developing breast cancer respectively.

To assess the robustness of our findings, re-analysis of the relationship between MTHFR C677T genetic polymorphism and breast cancer was done by changing the boundaries of the age categories. The same statistical significant differences persisted when the age boundaries were changed to " $35-60$ " years $\left(\mathrm{OR}_{\mathrm{TT}+\mathrm{CT} \text { vs } \mathrm{CC}}=1.9 ; 95 \%\right.$ $\mathrm{CI}(1.2-3.4) ; \mathrm{p}=0.01)$ and " $40-65$ " years $\left(\mathrm{OR}_{\mathrm{TT}+\mathrm{CT} \text { vs } \mathrm{CC}}=\right.$ $1.8 ; 95 \%$ CI $(1.1-3.0) ; \mathrm{p}$ value $=0.03)$.

However, no significant difference was found in the 1298 SNP between breast cancer cases and controls (Table 1). The allelic distribution of the two SNPs were in HardyWeinberg equilibrium $(\mathrm{p}>0.05)$.

Four different haplotypes appeared in our analysis CA, CC, TA and TC. The most frequent haplotypes was CA (677C-1298A) (cases: 38.3\%; controls: $45.4 \%$ ), while the rare haplotype was TC (cases: 3.6\%; controls: $8.3 \%$ ). Our results indicated that the two loci 677 and 1298 show strong linkage disequilibrium (LD) between cases and fair LD in controls as reflected by D' value. Carriers of the TA (677T-1298A) haplotype were 1.6 times more likely to have breast cancer (Table 2).
Table 2. Haplotype Frequencies of MTHFR among Breast Cancer Patients and Controls

\begin{tabular}{lrcll}
\hline $\begin{array}{l}\text { Haplotype } \\
677-1298\end{array}$ & Cases (\%) & Control (\%) & P value & OR (95\% CI) \\
\hline CA & $112(38.3)$ & $119(45.4)$ & & reference \\
CC & $84(28.9)$ & $66(25.2)$ & 0.8 & $1.1(0.71-1.6)$ \\
TA & $85(29.2)$ & $55(21)$ & 0.02 & $1.6(1.1-2.5)$ \\
TC & $11(3.6)$ & $22(8.3)$ & 0.1 & $0.5(0.25-1.15)$ \\
\hline
\end{tabular}

\section{Discussion}

Studying the genes present in the folate pathway and its polymorphism will help to determine people who are most susceptible of being affected by breast cancer and maybe will help in early detection and treatment of the disease.

In the United States of America a sudden increase in the probability of breast cancer was found after the age of 39 years $(0.49 \%$ to $3.76 \%)$. Another sudden increase was observed at the age of 70 years (3.53\% to $6.58 \%$ ) (American Cancer Society, 2012). In current study we divide our recruits at 40 and 60 years boundaries. We did not divide the recruits at 70 years because of the limited number of recruits above 70 years. Current study indicated that Jordanian women between the age of 40 and 60 years and who carry the MTHFR677TT genotype are at higher risk for the development of breast cancer. The findings persisted even when the age boundaries were changed to 40-65 years or to 35-60 years reflecting sturdiness of findings. Other age categories did not reveal similar trend.

A number of genetics and environmental risk factors contribute to the development of breast cancer. Familial breast cancer accounts for 6-12\% of total cases of breast cancer (Collaborative Group on Hormonal Factors in Breast Cancer, 2001). Genetic changes in BRCA1 and BRCA2 are strongly and vividly associated with familial breast cancer (Welcsh and King, 2001). Breast cancers associated with BRCA1 and BRCA2 mutations tend to develop at younger ages than sporadic breast cancers (National cancer institute, 2014). As such, it is perceivable that breast cancer for those $<40$ years is attributable to stronger familial factors other than MTHFR genetic polymorphism.

Other non genetic risk factors including aging, cosmic rays, exposure to deodorant, physical inactivity, BMI and western diet contributes to the development of breast 
cancer (Eliassen et al., 2006; Siegel et al., 2012; Marie A. et al., 2013; Kruk, 2014). It is expected that the longer exposure to such factors the higher the probability of breast cancer. Such anticipation may explain the inability of current study to find an association between MTHFR and breast cancer in patients older than 60 years.

The association of 677TT and breast cancer has been described previously (Zhang et al., 2010). The recent metaanalysis of 40 studies of 15260 cases and 20411 controls showed similar results that TT genotype of C677T is a risk factor for breast cancer [(TT vs CC: OR=1.2,95\% CI (1.1-1.3)]. Stratifying by ethnicity, significantly increased risk was only found in East Asians (Zhong et al., 2014). Additionally, in a meta-analysis on Asian population, the $\mathrm{T}$ allele and TT genotype was also a risk factor for breast cancer [(TT vs CC: $\mathrm{OR}=1.4,95 \% \mathrm{CI}=(1.2-1.6) ; \mathrm{p}=0.0003]$ (Rai, 2014b).

The distribution of A1298C MTHFR genetic polymorphism was not significantly different between the Jordanian with breast cases and control in all ages categories investigated. The findings were consistent independent of the genetic model adopted.

The finding of a meta-analysis that was conducted in 2006 and included 9044 subjects from 18 case-control studies that were conducted in Asian, and Caucasians population, revealed no difference in all genotype contrasts of MTHFR A1298C among cases and control (Zintzaras, 2006). Similarly, a more recent systematic review and meta-analysis of 29 studies compared 8649 breast cancer cases with 18672 age-matched controls, was not able to find any association between MTHFR A1298C and the risk of development of breast cancer (Jiao and Li, 2013). The latest meta-analysis on 33 casecontrol studies and contain 15919 breast cancer patients and 19700 control had the same conclusion (Rai, 2014a).

Individual MTHFR genetic polymorphisms might not act independently to affect the susceptibility to breast cancer (Zhong et al., 2014). The genotypes of adjacent SNPs are often highly correlated, that means they are in linkage disequilibrium and the interaction of the SNPs within haplotypes may be a major determinant of disease susceptibility comparing with the single polymorphisms (Zintzaras and Lau, 2008). Many studies report that the analysis of haplotype was more powerful than single polymorphism analysis (Zintzaras et al., 2006; Zintzaras and Lau, 2008). Hence, the investigation of the association between MTHFR haplotypes and breast cancer risk would give more sensitive information than that of individual polymorphisms.

In our study, strong association (linkage disequilibrium) between C677T and A1298C in MTHFR gene among cases was found $\left(D^{\prime}=0.657\right)$. Our results shows that there is a significant difference between cases and controls for those carriers of TA haplotype, for which they are 1.6 times at higher risk for breast cancer $(\mathrm{p}=0.024)$. A metaanalysis indicated that haplotype TC (677T-1298C) was significantly associated with increased breast cancer risk when compared with wild haplotype CA (677C-1298A), which was even more obvious than the comparison between 677 allele $\mathrm{T}$ and $\mathrm{C}$, and haplotype 677C-1298C could grant higher protection than 677C-1298A in East
Asians (Zhong et al., 2014). These results were consistent with other studies on combined genotypes analyses (Zintzaras et al., 2006).

We found that $677 \mathrm{CT}$ genetic polymorphism increases the risk of breast cancer in contrast to $\mathrm{CC}$ genotype (OR= 1.6; $95 \%$ CI $(0.99-2.6) ; \mathrm{p}=0.06)$. The same trend was observed in two other studies from Iran and Morocco (Hosseini et al., 2011; Diakite et al., 2012). The findings are difficult to explain as 677CT increases the risk of breast cancer, but neither TT nor CC genotypes.

The study findings are limited by relatively small sample size and lack of matching between cases and controls on other variables other than age. Additionally, the interacting effect of folate intake and/or folate serum level on the influence of MTHFR genetic polymorphism on breast cancer was not adequately collected or not measured at all. No reliable valid method was developed to assess patients' dietary intake, specifically, folate intake. A complicating issue is the fact that bread in Jordan is fortified with iron and folic acid under the auspices of ministry of health and the vast majority of Jordanians have daily intake of bread (Food Fortification Initiative, 2014). Moreover, healthy recruits were not tested or scanned to confirm that they are cancer-free at the time of recruitment, and were not followed up later to assure that they are still cancer-free. Finally, other genes may be involved in the modulation of breast cancer risk like TS (Luo et al., 2011).

In conclusion, the findings of the current study suggest that genetic polymorphism of MTHFR at C677T and its haplotype analysis at 677 and 1298 modulates the risk of breast cancer in the Jordanian population. To the best of our knowledge, no other study has examined the role of MTHFR (C677T and A1298C) genetic polymorphism and their haplotypes in the modulation of breast cancer in the Jordanian population and in the Middle East.

\section{Acknowledgements}

This study was supported by unconditional grant from the Deanship of Scientific Research (University of Jordan, Jordan). The study sponsors had no part in the study design, data collection, data analysis, data interpretations or in the writing of the manuscript. The sponsors had no role in the decision to submit the paper for publication.

\section{References}

American Cancer Society. 2012. Cancer Facts and Figures 2012 [Online]. Available: http://www.cancer.org/acs/groups/ content/@epidemiologysurveilance/documents/document/ acspc-031941.pdf [Accessed 25-3-2015 2015].

Butterworth AS, Higgins JP, Pharoah P (2006). Relative and absolute risk of colorectal cancer for individuals with a family history: a meta-analysis. Eur J Cancer, 42, 216-27.

Chad M. Barnett, Laura Boehnke Michaud, Francisco J. Esteva (2014). Bresat cancer. In 'Pharmacotherapy: A Pathophysiologic Approach', Eds Mc Graw Hill education,

Cochran G (1954). Some methods for strengthening the common $\chi$ tests. Biometrics, 10.

Collaborative Group on Hormonal Factors in Breast Cancer (2001). Familial breast cancer: collaborative reanalysis of individual data from 52 epidemiological studies including 
58,209 women with breast cancer and 101,986 women without the disease. Lancet, 358, 1389-99.

Diakite B, Tazzite A, Hamzi K, et al (2012). Methylenetetrahydrofolate reductase C677T polymorphism and breast cancer risk in Moroccan women. African Health Sciences, 12, 204-9.

Eliassen AH, Colditz GA, Rosner B, et al (2006). Adult weight change and risk of postmenopausal breast cancer. JAMA, 296, 193-201.

Food Fortification Initiative. 2014. Middle East - Fortification Status [Online]. Available: http://www.ffinetwork.org/ regional_activity/middle_east.php\#middle_east.php?s=2\&_ suid $=142601829808809411473741271781$ [Accessed 10-3-2015 2015].

Foulkes WD (2008). Inherited susceptibility to common cancers. $N$ Eng J Med, 359, 2143-53.

Frosst P, Blom HJ, Milos R, et al (1995). A candidate genetic risk factor for vascular disease: a common mutation in methylenetetrahydrofolate reductase. Nature Genetics, $\mathbf{1 0}$, 111-3.

Gaughan DJ, Barbaux S, Kluijtmans LA, et al (2000). The human and mouse methylenetetrahydrofolate reductase (MTHFR) genes: genomic organization, mRNA structure and linkage to the CLCN6 gene. Gene, 257, 279-89.

Hosseini M, Houshmand M, Ebrahimi A (2011). MTHFR polymorphisms and breast cancer risk. Arch Medical Science, 7, 134-7.

Jiang Y, Hou J, Zhang Q, et al (2013). The MTHFR C677T polymorphism and risk of acute lymphoblastic leukemia: an updated meta-analysis based on 37 case-control studies. Asian Pac J Cancer Prev, 14, 6357-62.

Jiao Z, Li D (2013). Lack of association between MHTFR Glu429Ala polymorphism and breast cancer susceptibility: a systematic review and meta-analysis of 29 research studies. Tumour Biology, 34, 1225-33.

Jordan ministry of health. 2010. Annual Incidence of cancer in Jordan [Online]. Available: http://www.moh.gov.jo/EN/ Pages/Periodic-Newsletters.aspx [Accessed 21-1-2015 2015].

Kruk J (2014). Lifestyle Components and Primary Breast Cancer Prevention. Asian Pac J Cancer Prev, 15, 10543-55.

Liew S, Gupta ED (2015). Methylenetetrahydrofolate reductase (MTHFR) C677T polymorphism: Epidemiology, metabolism and the associated diseases. Eur J Medical Genetics, 58, 1-10.

Luo B, Repalli J, Yousef AM, et al (2011). Human thymidylate synthase with loop 181-197 stabilized in an inactive conformation: ligand interactions, phosphorylation, and inhibition profiles. Protein Science, 20, 87-94.

Marie A., Barbara G., Terry L., et al 2013. Pharmacotherapy Principles and Practice.

National cancer institute. 2014. BRCA1 and BRCA2: Cancer Risk and Genetic Testing [Online]. Available: http://www. cancer.gov/cancertopics/genetics/brca-fact-sheet [Accessed 6-3-2015 2015].

Ozen F, Sen M, Ozdemir O (2014). Methylenetetrahydrofolate reductase gene germ-line C677T and A1298C SNPs are associated with colorectal cancer risk in the Turkish population. Asian Pac J Cancer Prev, 15, 7731-5.

Pharoah PD, Day NE, Duffy S, et al (1997). Family history and the risk of breast cancer: a systematic review and metaanalysis. Intl J Cancer, 71, 800-9.

Rai V (2014a). Methylenetetrahydrofolate Reductase A1298C Polymorphism and Breast Cancer Risk: A Meta-analysis of 33 Studies. Ann Medical Health Sciences Res, 4, 841-51.

Rai V (2014b). The methylenetetrahydrofolate reductase C677T polymorphism and breast cancer risk in Asian populations.
Asian Pac J Cancer Prev, 15, 5853-60.

Rodriguez S, Gaunt TR, Day IN (2009). Hardy-Weinberg equilibrium testing of biological ascertainment for Mendelian randomization studies. Am J Epidemiol, 169, 505-14.

Shiovitz S, Korde LA (2015). Genetics of breast cancer: a topic in Evolution. Ann Oncol. [Epub ahead of print]

Siegel R, Naishadham D, Jemal A (2012). Cancer statistics, 2012. CA: a Cancer J Clin, 62, 10-29.

Walker SE, Lorsch J (2013). Sanger dideoxy sequencing of DNA. Method Enzymol, 529, 171-84.

Welcsh PL, King MC (2001). BRCA1 and BRCA2 and the genetics of breast and ovarian cancer. Human Molecular Genetics, 10, 705-13.

Xu X, Gammon MD, Zeisel SH, et al (2008). Choline metabolism and risk of breast cancer in a population-based study. FASEB $J, 22,2045-52$.

Yang YB, Shang YH, Tan YL, et al (2014). Methylenetetrahydrofolate reductase polymorphisms and susceptibility to esophageal cancer in Chinese populations: a meta-analysis. Asian Pac J Cancer Prev, 15, 1345-9.

Yousef AM, Shomaf M, Berger S, et al (2013). Allele and genotype frequencies of the polymorphic methylenetetrahydrofolate reductase and colorectal cancer among Jordanian population. Asian Pac J Cancer Prev, 14, 4559-65.

Zhang, Qiu LX, Wang ZH, et al (2010). MTHFR C677T polymorphism associated with breast cancer susceptibility: a meta-analysis involving 15,260 cases and 20,411 controls. Breast Cancer Res Treatment, 123, 549-55.

Zhong S, Chen Z, Yu X, et al (2014). A meta-analysis of genotypes and haplotypes of methylenetetrahydrofolate reductase gene polymorphisms in breast cancer. Molec Cell Biochem, 41, 5775-85.

Zintzaras E (2006). Methylenetetrahydrofolate reductase gene and susceptibility to breast cancer: a meta-analysis. Clinical Genetics, 69, 327-36.

Zintzaras E, Koufakis T,Ziakas PD, et al (2006). A meta-analysis of genotypes and haplotypes of methylenetetrahydrofolate reductase gene polymorphisms in acute lymphoblastic leukemia. Eur J Epidemiol, 21, 501-10.

Zintzaras E, Lau J (2008). Synthesis of genetic association studies for pertinent gene-disease associations requires appropriate methodological and statistical approaches. $J$ Clin Epidemiol, 61, 634-45. 